EPJ Web of Conferences 19, 06002 (2012)

DOI: $10.1051 /$ epjconf/20121906002

(C) Owned by the authors, published by EDP Sciences, 2012

\title{
The chemical evolution of the Galactic Bulge seen through micro-lensing events
}

\author{
S. Feltzing ${ }^{1, a}$, T. Bensby ${ }^{1}$, J. Meléndez ${ }^{2}$, D. Adén ${ }^{1}$, M. Asplund ${ }^{3}$, A. Gould ${ }^{4}$, \\ J. Johnson ${ }^{4}$, S. Lucatello ${ }^{5}$ and A. Gal-Yam ${ }^{6}$ \\ 1 Lund Observatory, Box 43, 22100 Lund, Sweden \\ 2 Universidade de São Paulo, IAG, Rua do Matão 1226, Cidade Universitária, \\ São Paulo 05508-900, Brazil \\ 3 Research School of Astronomy and Astrophysics, Australian National University, \\ Cotter Rd., Weston, ACT 2611, Australia \\ 4 Department of Astronomy, Ohio State University, 140 W. 18th Avenue, Columbus, \\ $\mathrm{OH}$ 43210, USA \\ 5 Osservatorio Astronomico di Padova, Vicolo dell'Osservatorio 5, 35122 Padua, Italy \\ ${ }^{6}$ Benoziyo Center for Astrophysics, Faculty of Physics, The Weizmann Institute of Science, \\ Rehovot 76100, Israel
}

\begin{abstract}
Galactic bulges are central to understanding galaxy formation and evolution. Here we report on recent studies using micro-lensing events to obtain spectra of high resolution and moderately high signalto-noise ratios of dwarf stars in the Galactic bulge. Normally this is not feasible for the faint turn-off stars in the Galactic bulge, but micro-lensing offers this possibility. Elemental abundance trends in the Galactic bulge as traced by dwarf stars are very similar to those seen for dwarf stars in the solar neighbourhood. We discuss the implications of the ages and metallicity distribution function derived for the micro-lensed dwarf stars in the Galactic bulge.
\end{abstract}

\section{INTRODUCTION}

Galactic bulges are emerging as inherently complex features in spiral galaxies. Numerous studies have shown them to have several spatial structures overlaying each other. The Milky Way is no different over the last decades our view of the stellar content, gas and stellar dynamics in the inner few kpc of our own galaxy has developed significantly. If the spatial structures are uniquely related to dynamical and chemical features is a very actively studied field.

New surveys such as VISTA Variables in The Via Lactea (VVV) public survey (described in [21]) are looking deeper and deeper in to this intriguing Galactic component. Several outstanding questions remain un-resolved including the true shape of the metallicity distribution function (MDF) and how the MDF is connected to different spatial and kinematical structures. A recent discussion of these issues can be found in [4]. Other questions concern the star formation history of the bulge and the presence or absence of age spreads as, for example, traced by asymptotic giant stars [24].

The history of a stellar population is imprinted in its stars. The elemental abundances in the atmospheres of stars often remain unperturbed over time and act as time-capsules showing the mixture of elements present in the gas from which the stars formed. This is in particular true for dwarf stars and their

\footnotetext{
a e-mail: sofia@astro.lu.se
}

This is an Open Access article distributed under the terms of the Creative Commons Attribution-Noncommercial License 3.0, which permits unrestricted use, distribution, and reproduction in any noncommercial medium, provided the original work is properly cited. 
spectra, even for metal-rich stars, are fairly straightforward to analyze and are the best tracers of galactic chemical evolution [11]. However, dwarf stars in the Galactic bulge are too faint to be observed under normal circumstances $(V=19-20$, as seen in for example colour-magnitude diagrams obtained with the HST, [12]). The chemical history of the bulge has therefore mainly been studied using intrinsically bright giant stars. Results based on giant spectra are not trivial to interpret as evolutionary processes erase some of the abundance information and the cool atmospheres of giants, rich in molecules, are difficult to analyse, see discussion in [13]. IR spectroscopy of bulge giants has recently become feasible but is still limited by the very restricted wavelength coverage on existing spectrographs. A recent example is given by the CRIRES spectra analysed in [20]. However, the underlying assumption that giants accurately represent all stars has not yet been rigorously tested, as discussed in [22]. Therefore, a metallicity distribution function based on red giant star may not reflect the original distribution. Physical processes within the red giant stars can also lead to the erasure of some of the original abundance signatures. This is in particular the case for $\mathrm{C}, \mathrm{N}$, and Li. These abundances are eventually altered in all red giants and in some stars $\mathrm{O}, \mathrm{Na}, \mathrm{Mg}$, and $\mathrm{Al}$ are also altered as discussed in [17]. This means that a true study of the star formation history in the Galactic bulge requires the study of dwarf stars. Furthermore, the precision achieved in dwarfs is better than in giants, allowing us to look for any substructure that may be present in the bulge population. Finally, for dwarf stars close to the turn-off point or on the sub-giant branch it is possible to derive individual ages. These give us a unique insight in to the age structure of the Galactic bulge.

Micro-lensing offers the unique opportunity to observe dwarf stars in the bulge. When the star is lensed by a foreground object its magnitude can increase more than 5 magnitudes making it possible to obtain a spectrum of high resolution and $\mathrm{S} / \mathrm{N}$ so that a standard abundance analysis can be done [7].

The observed micro-lensing events discussed in this contribution and their analysis are fully documented in [6] and [7]. Issues concerning limb-darkening are further developed in [16].

\section{MICRO-LENSED DWARF STARS IN THE GALACTIC BULGE - DISCUSSION}

\subsection{Abundance trends}

We find that the elemental abundance trends in the Galactic bulge as traced by the micro-lensed dwarf stars is very similar, if not identical, to that found in the solar neighbourhood for dwarf stars with kinematics typical of the thick disk [7]. Recent studies of red giant stars have also shown great similarities between the local thick disk giants and the giants in the Galactic bulge [1]. Thus it appears that the earlier results where red giant stars in the Galactic bulge showed large $\alpha$-enhancements also at solar and even super-solar metallicities must be ascribed to the difficulty in analysing optical spectra of metal-rich red giants (see also discussion in [6]).

\subsection{MDF and IMF}

In our two papers [6] and [7] we compare the MDF based on the small number of micro-lensed dwarf stars available with the then best MDF based on spectroscopy of red giant stars as analysed in [26]. We found a significant difference with the dwarf stars showing a bi-modal MDF. A recent re-analysis of the spectra of these red giant stars has changed the situation somewhat by making the MDF based on giant stars somewhat more bi-modal. This re-analysis is presented in [14]. A KS-test between that result and the MDF based on micro-lensed dwarf stars (including only those presented by us in [7]) is inconclusive. The MDFs could be drawn from the same population or not.

The most recent update of our MDF based on micro-lensed dwarf stars, in total 37 stars (end of September 2011), still shows a bi-modal MDF and with an ever increasing fraction of metal-rich stars.

An interesting implication of the true shape of the MDF concerns the Initial Mass Function (IMF). The origin of the slope of the IMF is much debated (for a short, recent introduction to the debate see 


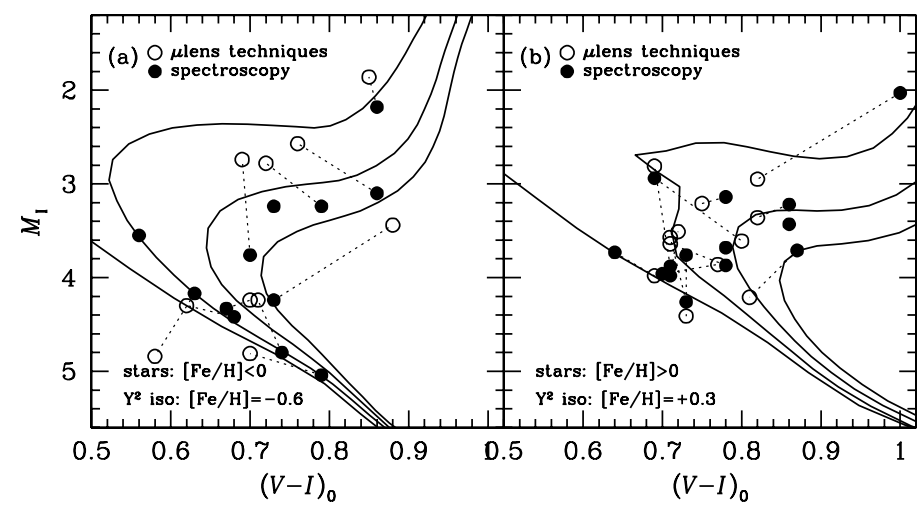

Figure 1. Colour-magnitude diagrams for the micro-lensed dwarf stars. The colours and magnitudes are determined using micro-lensing techniques $(\circ)$ and spectroscopy $(\bullet)$. a Shows the stars with $[\mathrm{Fe} / \mathrm{H}]<0$ and $\mathbf{b}$ the stars with $[\mathrm{Fe} / \mathrm{H}]>0$. Each colour-magnitude diagram also show representative $Y^{2}$ isochrones for $1,5,10$, and $15 \mathrm{Gyr}$, from [10]. For each star we connect the result based on micro-lensing techniques with that based on spectroscopy using a dotted line. 4 stars have no values from micro-lensing techniques (see [7]).

[18]). Many processes leads to roughly the same slope and there is not much evidence that metallicity influences the shape or slope of the IMF, a review can be found in [3]. Recent work on the IMF in the Galactic bulge has resulted in interesting conclusions. The peak of the MDF depends on the slope of the IMF. The chemical evolution model of the Galactic bulge by [2] is based on the photometric MDF of giant stars by [25] and on the spectroscopic MDF of giant stars by [13]. In [2] an IMF much flatter than in the solar neighbourhood is found, i.e., an IMF skewed towards high mass stars.

A more recent model used the spectroscopic MDF, still using the original results from [26], also requires a flat IMF to reproduce the peak of the MDF as derived from the red giant stars ([5]). However, the MDF based on micro-lensed dwarf stars persistently shows two well-defined peaks, one that can be associated with a metal-poor old bulge, and another with super-solar metallicities that can be associated with a younger population (compare also the discussion of the connection between the stellar kinematics and their metallicities presented in [4]). Hence, the IMF no longer has to be flat to explain a single peaked solar metallicity MDF that was made in $0.5 \mathrm{Gyr}$, as in these recent models ([5]). To reproduce the bi-modal nature of the bulge MDF, probably a normal IMF can be used for the metal-poor bulge, while contributions from type Ia SN might explain the younger metal-rich peak. However, more detailed models are needed before firm conclusions can be drawn.

\subsection{Ages}

Dwarfs near the turn-off are unique as we can get stellar ages for them. Figures 1 and 2 show a summary of results of our determination of stellar parameters and ages for stars presented in [7]. In Fig. 1 we split the stars according to metallicity. We show two different values of $M_{\mathrm{I}}$ and $(V-I)_{0}$ for each star. One is based on the effective temperature and surface gravity derived from the stellar spectra alone (spectroscopic values). The micro-lensing technique relates the magnitude of the star to that of the red clump stars in the same field. The advantages and draw-backs of each technique is discussed in our recent paper, [7]. The thing to take away from the left-hand panels in Fig. 1 is that regardless of which technique is used, stars with sub-solar metallicities essentially trace an old turn-off, while stars with super-solar metallicities show a wider range of ages. This is still true when the latest events are included (Bensby et al. 2012 in prep.).

A surprising result from the micro-lensed dwarf stars is the presence of a large age spread among the most metal-rich stars. This result might appear unexpected given the large amount of evidence based on 


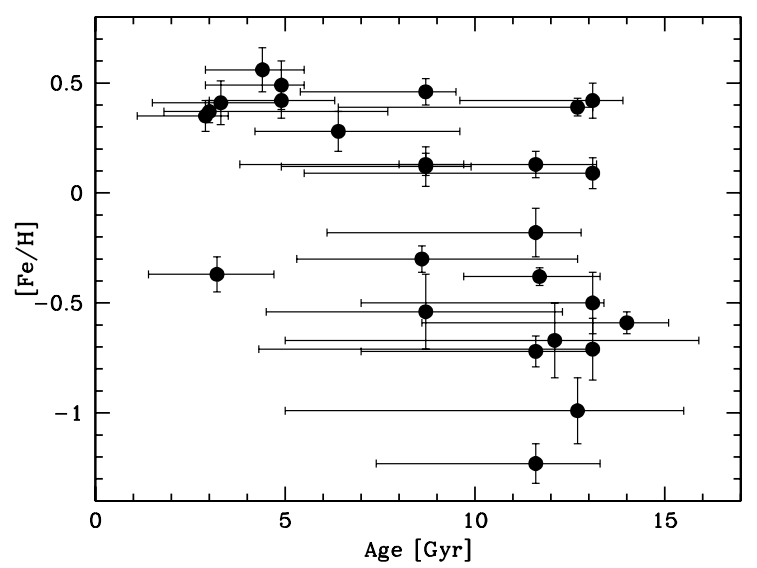

Figure 2. The ages and metallicities of the 26 dwarf stars as derived in [7].

deep CMDs that show a red and faint turn-off, most often interpreted as the result of a uniquely old and metal-rich stellar population (as shown in numerous studies, including [8, 12, 15, 19, 25]). However, there is evidence from AGB stars of an intermediate age population in the Galactic bulge. This has been seen at least in three independent studies $([9,23,24])$.

Based on ISOGAL and DENIS data of the inner $10^{\circ}$ of the Galactic bulge [24] find a few hundred asymptotic giant branch stars, which is consistent with their inferences from the near infrared CMDs and [23] find evidence for Tc in a sub-sample of their C-stars, indicative of third dredge up and a minimum stellar mass of $1.5 \mathrm{M}_{\odot}$ which implies an upper age limit of $3 \mathrm{Gyr}$. Our data for micro-lensed dwarfs appear to confirm the existence of such an intermediate age population in the inner kpcs.

\section{SUMMARY AND OUTLOOK}

So far we have presented elemental abundances and ages for 26 micro-lensed dwarf stars ([6] and [7]). They show that dwarf stars in the Galactic bulge share the elemental abundance trends with the thick disk in the solar neighbourhood and have an MDF that is bi-modal. This is also true for the most recent observations (Bensby et al. 2012 in prep.). Surprisingly, we find, among the stars with super-solar metallicities, a wide range of ages. This remains to be better explained, but we note that AGB stars and variable stars (Miras) present in the Galactic bulge also point to a sub-population with an intermediate age. We note that this is, on the surface, contradictory to the red and faint turn-offs seen in all CMDs of the Bulge. However, a smaller intermediate age, metal-rich stellar population can most likely still be accommodated. Detailed modelling of this and larger samples of both dwarf and giant stars in the Galactic bulge covering wider areas of the bulge are needed to fully understand the connection between the various spatial structures in the bulge and the MDF.

\section{References}

[1] Alves-Brito, A., Meléndez, J., Asplund, M. et al., A\&A 513, (2010) A35

[2] Ballero, S.K., Matteucci, F., Origlia, L. \& Rich, R.M. A\&A 467, (2007) 123

[3] Bastian, N., C., Kevin, R. \& Meyer, M.R., ARA\&A 48, (2010) 339

[4] Babusiaux, C., Gómez, A., Hill, V., et al. A\&A 519, (2010) A77

[5] Cescutti, G. \& Matteucci, M., A\&A 525, (2011) A126

[6] Bensby, T., Feltzing, S., Johnson, J.A., et al., A\&A 512, (2010) A41

[7] Bensby, T., Adén, D., Meléndez, J., Gould, A., et al., A\&A 533, (2011) A134 
[8] Clarkson, W.I., Sahu, K.C., Anderson, J., et al. ApJ 735, (2011) 37

[9] Cole, A.A. \& Weinberg, M.D., ApJ 574, (2002) L43

[10] Demarque, P., Woo, J.-H., Kim, Y.-C. \& Yi, S. K., ApJS 155, (2004) 667

[11] Edvardsson, B., Andersen, J., Gustafsson, B., et al., A\&A 275, (1993) 101

[12] Feltzing, S. \& Gilmore, G., A\&A 355, (2000) 949

[13] Fulbright, . P., McWilliam, A., \& Rich, R.M., ApJ 636, (2006) 821

[14] Hill, V., Lecureur, A., Gómez, A., et al., A\&A 534, (2011) A80

[15] Holtzman, J.A., Light, R.M., Baum, W.A., et al., AJ 106, (1993) 1826

[16] Johnson, J.A., Subo, D. \& Gould, A., ApJ 713, (2010) 713

[17] Kraft, R., ApJ 104, (1992) 645

[18] Oey, M.S., ApJ Letters 739, (2011) L46

[19] Ortolani, S., Renzini, A., Gilmozzi, R., et al., Nature 377, (1995) 701

[20] Ryde, N., Gustafsson, B., Edvardsson, B., et al., A\&A 509, (2010) A20

[21] Saito, R., Hempel, M., Alonso-García, J., et al., Messenger 141, (2010) 28

[22] Taylor, B.J. \& Croxall, K., MNRAS 357, (2005) 967

[23] Uttenthaler, S., Hron, J., Lebzelterm T., et al., A\&A 463, (2007) 251

[24] van Loon, J.Th., Gilmore, G.F., Omont, A., et al. MNRAS 338, (2003) 857

[25] Zoccali, M., Renzini, A., Ortolani, S., et al., A\&A 399, (2003) 931

[26] Zoccali, M., Hill, V., Lecureur, A., et al., A\&A 486, (2008) 177 